\title{
Wnt5a Gene Plays a Role in Mouse Embryonic Orofacial Development
}

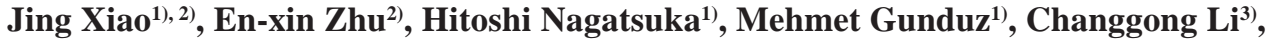 \\ Parviz Minoo $^{3)}$ and Noriyuki Nagai ${ }^{1)}$
}

\begin{abstract}
${ }^{1)}$ Department of Oral Pathology, Graduate School of Medicine, Dentistry and Pharmaceutical Sciences, Okayama University, Japan ${ }^{2}$ Department of Oral Pathology, College of Stomatology, Dalian Medical University, China

${ }^{3}$ Department of Pediatrics, Keck School of Medicine, University of Southern California, the U.S.A. (Accepted for publication 20 January, 2006)
\end{abstract}

\begin{abstract}
To determine if Wnt5a gene involved in orofacial development, especially secondary palate development, we checked Wnt5a protein and mRNA in wild-type mouse embryos, and also searched Wnt5a knockout mice palates. We found Wnt5a is expressed during orofacial development, especially necessary for normal secondary palate and tongue morphogenesis. Wnt5a deficient mice exhibit cleft palate and abnormal distortion tongue suggests Wnt5a is essential to the palate development. Our data suggested that defection of MEE proliferation in Wnt5a (-/-) secondary palate indicates Wnt5a gene may regulate palate growth.
\end{abstract}

Key words:Wnt5a, Development, Palate, Gene, KO mouse

\section{Introduction}

Wnt5a a member of WNT family of signaling molecules though the non-canonical $\mathrm{Wnt} / \mathrm{Ca} 2+$ pathway was shown to underlie the P-D outgrowth of limb and distal airway development of lung. Wnt5a null mice was created by Dr. McMahon and his co-workers and published on the journal of Development (Yamaguchi et al., 1999). The targeted allele is likely a null since the gene is disrupted in exon 2, at codon 31 of the Wnt5a protein. Homozygous mutant offspring of heterozygous intercrosses were born but died shortly thereafter within two hours, and showed under-growth of snout, limbs and tail. Truncation of proximal skeleton and absence of distal digits of limbs was reported to correlate with reduced proliferation of putative progenitor cells within the progress zone. In Wnt5a knockout lung studies, Dr. Li (Li et al., 2002) also found the phenotype of the trachea from Wnt5aKO lungs is foreshortened with reduced number of cartilage rings, and the ratio of proliferating cells over the total number of cells is increased in Wnt5aKO lungs when compared with the wild type controls. A striking phenotype of the Wnt5aKO lungs at E18 later embryonic stage of development is the hypercellular and thickened interstitium (inter-saccular walls).

The most common of human birth defects is cleft lip and/or palate, a complex trait caused by multiple genetic and environmental factors. Cleft palate may result from disturbances at any stage of palate development: Defective palatal shelf growth, delayed or failed shelf elevation, defective shelf fusion, failure of medial edge epithelium cell death, post-fusion rupture and failure of mesenchymal consolidation and differentiation. Growth of the palate shelves depends on the survival and continued proliferation of mesenchymal cells that originate from neural crest and mesodermal cells of the first branchial arch, and the epitheliummesenchyme interaction regulated by various signaling molecules is essential to the growth process of palatal shelves. Studies by using transgenic animal models provided details of the interactions between the epithelium and mesenchyme that lead to palate growth and development, such as Fgf10 is expressed in the mesenchyme, then activates its receptor, FGF receptor $2 \mathrm{~b}$ (Fgfr2b), which is

Corresponding to:Jing Xiao, Department of Oral Pathology, College of Stomatology, Dalian Medical University, China located in the epithelium. Finally, Fgfr2b mediates expression of sonic hedgehog (Shh) in the epithelium.

\section{Materials and Methods}

RNA extraction and Semi-quantitative RT-PCR

Total RNA was isolated by using Trizol (GIBCO). AMV Reverse Transcriptase First-strand cDNA Synthesis Kit (Life Sciences Inc., Japan) was used to generated cDNA. Sequences of the primers of the genes are as follows. Mouse Wnt5a: 5'CTTCGCCCAGGTTGTTATAGAAGC-3' (forward), 5'CTGCCAAAGACAGAAGTATTGTCC-3' (backward).

\section{In situ hybridization}

Whole-mount in situ hybridization was performed as described by Xiao (2003) with the following modifications. In brief, RNA anti-sense and sense probes of Wnt5a were prepared with digoxigenin as label and a 1.1-kb cDNA of Wnt5a 3'-UTR as template. E10 mouse embryo as well as E 11.5 and E13.5 mandible and tongue were fixed with $4 \%$ paraformaldehyde (PFA) in calcium/magnesium-free DEPC-PBS at $4^{\circ} \mathrm{C}$.

\section{Immunohistochemistry}

Five micrometer (ìm)-tissue sections were prepared. Subsequent to deparaffinization, the sections were hydrated, heated in $10 \mathrm{mM}$ citrate buffer (pH6.0) and treated with 1\% H2O2 in methanol for $10 \mathrm{~min}$ and blocked with $10 \%$ of normal serum. The sections were then incubated with primary antibodies at 40C overnight. Biotinylated secondary antibody and streptavidin-peroxidase conjugate (Vector Laboratories, Inc.) were used to detect the bound antibodies. The sections were developed with diaminobenzidine. WNT5A primary antibody was purchased from R\&D Systems.

\section{Proliferation assay}

Pregnant female mice were injected intraperitoneally with bromodeoxyuridine (BrdU) reagent at 100 il per $10 \mathrm{~g}$ body weight. Ten minutes after injection, embryonic heads were dissected out, fixed, dehydrated and paraffin embedded. The sections were then rehydrated and treated with BrdU staining kit as recommended by the manufacturer (Zymed Laboratories Inc., U.S.). 


\section{Results and Discussion}

\section{Spatial and temporal distribution of Wnt5a gene in orofacial} development

Whole-mount in situ hybridization for Wnt5a demonstrated transcripts at the tip of various growing appendages, but particularly in the snout, mandible, limb and genital tubercle expressed as early as embryonic day E10 embryo (Fig1,A). Intense level of Wnt5a transcripts continued expressing in the mandible and tongue until E13.5 (Fig1, B). During precisely development stages of secondary palate, semi-quantitative RT-PCR of secondary palate and tongue showed higher level Wnt5a signaling compared with same stage lung. PCR results also showed intense level of Wnt5a expressed in first lower molar tooth germs in E16.5 of early bell stage (Fig1, C). Immunohistochemical staining of wild type orofacial tissues was performed in E13 and E14.5, which is the precisely stage of palate vertically rapid growth and horizontally fusion, respectively. In E13 wild type orofacial regions, there was high-level, diffused expression of WNT5a protein that appeared to be localized to both the epithelial and the mesenchymal compartments of secondary palate, tongue and mandible (Fig1, D). In E14.5, both abundance and spatial distribution of WNT5a become dynamic. At this stage, intensity of WNT5a staining in the nasal epithelium is increased compared with that of oral epithelium of secondary palate, and the reduced level of Wnt5a expressed in E14.5 palate mesenchyme than E13. In addition, we observed higher level Wnt5a protein located in both internal enamel epithelium and its adjunction dental mesenchyme of first molar (Fig1, E).

\section{Mutation in Wnt5a (-/-) secondary palate and tongue}

We analyses the secondary palate and tongue by making frontal series sections of E14.5 and E18.5 (-/-) mice heads. Comparison with control, all Wnt5a (-/-) mice (4 embryos at E14.5, 2 embryos at E15.5 and 2 embryos at E18.5) exhibited completely palate cleft. At the E14.5, Wnt5a (-/-) mice tongue showed abnormal distortion and could not drop into the oral cavity; Even though anterior portions of both side of palatal shelves elevated above the tongue, but keep separated without fusion. And the posterior portions still keep in the vertically growth beside of the tongue (Fig2, A). At the E18.5, the tongue and anterior portion of secondary palate showed the similar pattern as in E14.5, but the nasal epithelium of posterior portion became to fuse with nasal septum (Fig2, B).

\section{Proliferation analysis in Wnt5a (-/-) orofacial development}

We checked the proliferation ratio by performing BrdU staining in E14.5 Wnt5a (-/-) mice, no difference showed between the mesenchymal compartments of palate, as well as tongue and tooth germ. Except the medial edge epithelium (MEE) of E14.5 Wnt5a (-/-) palate which disappeared the proliferation completely, even though the palatal shelves keep separated without fusion (data no

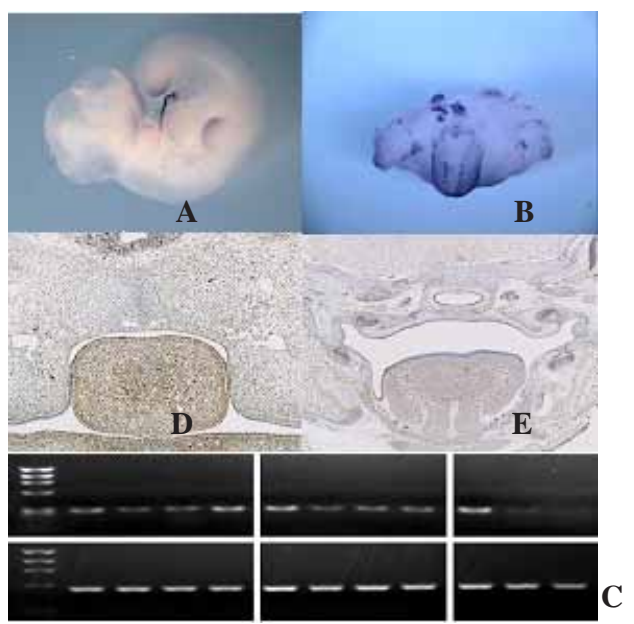

Fig. 1 Spatial and temporal distribution of Wnt5a gene in orofacial development

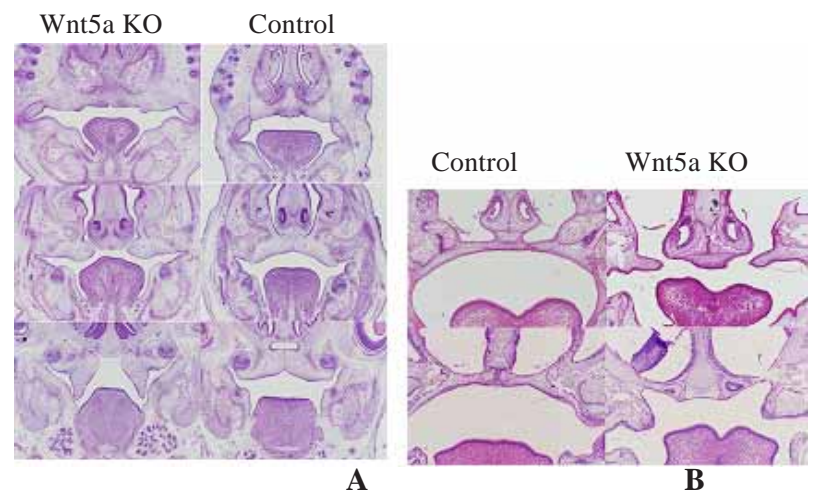

Fig.2 Mutation in Wnt5a (-/-) secondary palate and tongue

shown). The similar pattern of the AER (Apical ectodermal ridge) in limb development was reported, which the role of AER signals in regulating outgrowth and P-D patterning of limb (Yamaguchi et al., 1999).

\section{References}

1. Yamaguchi, T. P., Bradley, A., McMahon, A. P., and Jones, S. A. (1999). Wnt5a pathway underlies outgrowth of multiple structures in the vertebrate embryo. Development 126, 1211-1223.

2. Li, C., Xiao, J., Hormi, K., Borok, Z., Minoo, P. (2002).Wnt5a participates in distal lung morphogenesis. Dev Biol. 248, 68-81.

3. Xiao, J., Li, C., Zhu, N. L., Borok, Z., and Minoo, P. (2003). Timeless in lung morphogenesis. Dev Dyn. 228, 82-94. 